\title{
Existence of Financial Equilibria in a General Equilibrium Model with Piece-Wise Smooth Production Manifolds
}

\author{
Pascal Stiefenhofer \\ Brighton Business School, University of Brighton, Brighton, UK \\ Email: p.stiefenhofer@brighton.ac.uk
}

How to cite this paper: Stiefenhofer, P. (2017) Existence of Financial Equilibria in a General Equilibrium Model with Piece-Wise Smooth Production Manifolds. Journal of Mathematical Finance, 7, 671-681.

https://doi.org/10.4236/jmf.2017.73035

Received: April 22, 2017

Accepted: July 24, 2017

Published: July 27, 2017

Copyright $\odot 2017$ by author and Scientific Research Publishing Inc. This work is licensed under the Creative Commons Attribution International License (CC BY 4.0).

http://creativecommons.org/licenses/by/4.0/

\begin{abstract}
This paper considers a general equilibrium model with production and uncertainty. It is postulated that the firm finances its production capacity through the stock market and that its operational costs are covered through revenues. It is assumed that firms have linear technologies exhibiting constant returns to scale. Their production sets are piece-wise smooth convex manifolds. By method of regularization, it is shown using transversally theory that financial equilibria exists.
\end{abstract}

\section{Keywords}

Existence of Equilibria, Transversality, Linear Technology, General Equilibrium, Production, Regularization

\section{Introduction}

General equilibrium theory is the study of simultaneous interaction between market demand and market supply. It aims at explaing the functioning of markets as a whole. The basic model, known as the Arrow-Debreu model is represented in Debreu [1]. Its properties, such as the existence and efficiency of equilibria are well understood. However, these results are derived under a set of strong assumptions. For example, the model assumes a complete real asset structure and disregards any financial structure. This paper aims at moving towards a more realistic model that incorporates financial assets. Hence, a generalization of the private ownership Arrow-Debreu model to a model with financial assets is considered in this paper. Modelling the finance of production will lead to a complete contingent production model, where all production risks are covered. This, again, is not representing the real world satisfactorily. Hence, 
we assume that financial markets are incomplete. This implies that not all risk in the economy can be fully hedged. The aim of this paper is to develop such a model and to show that it is well defined.

The literature on general equilibrium modles with production mainly rests on the concepts introduced by Drèze [2] and Grossmann \& Hart [3] which require to assign some sort of utility function to the firm. The two concepts as applied to many models slightly differ in the choice of average utility utilized (average utility of initial/final share holders). For a sample of the huge literature applying these concepts see [1] [2] [4] [5] [6] and the references therein.

At variance with the above literature, this paper introduces a model of the firm, where its activity is independent of any average utility of the stock holders [7]. It postulates that firms maximize long run profits and make financial and real decisions sequentially over two periods. The assumption of long run profit maximization is justified by the sequential optimization structure of the firm introduced. Firms issue stocks in period one in order to acquire the cash needed to install production capacity. The optimal quantity of stocks issued by each firm is endogenously determined by the model. Once capacity is installed, after uncertain state of nature has occurred at the beginning of period two, firms produce real goods subject to capacity and technological constraints. The ownership structure introduced in this model eliminates the strategic choice problem of the firm present in the literature. Here, stock holders do not decide about the optimal input vector of the firm in period one. They invest in firms by purchasing stocks in order to transfer wealth across time and between uncertain states of nature. The total quantity of stocks demanded is equal to total quantity of stocks supplied by firms in the same period. The value of total stocks issued by a firm bounds the value of inputs a firm can purchase in period two. Real activities of the firm take place after uncertainty in period two has resolved. These production activities correspond to finding the optimal net activity vector at given prices and revealed state of the world such that profits are maximized at given production capacity.

Stiefenhofer [7] shows that for convex smooth production manifolds with an endogenized price and technology dependent real asset structure, which is transvers to the reduced rank Grassmanian manifolds, equilibrium exists generically in the endowments by the application of Thom's parametric transversality theorem. The paper generalizes this result to the non-smooth convex production set case, where the piecewise linear production manifolds are regularized by convolution. Existence then follows from the smooth case.

The model of the firm is introduced in Section 2. Section 3 shows generic existence for linear production technologies with convex piecewise smooth production manifolds. Section 4 is a conclusion.

\section{The Model of the Firm}

We consider a two period $t \in\{0,1\}$ model with uncertainty in period 1 represented as states of nature. An element in the set of mutually exclusive and 
exhaustive uncertain events is denoted $s \in S=\{1, \cdots, S\}$, where by convention $s=0$ represents the certain event in period 0 , and $S$ denotes the set of all mutually exclusive uncertain events. This set denotes the overall description of uncertainty in the model, which is characterized by idiosyncratic and aggregate risk. The general uncertainty space is described by the Cartesian product $S=\mathcal{S} \times \tilde{\mathcal{S}}$. For every production set $Y_{j}$, there exists a set of states of nature $S_{j}=\{1, \cdots, \mathbb{S}\}$, where $\mathbb{S} \geq 2$, for all $S_{j}$. Denote $\mathcal{S}=\left\{S_{1}, \cdots, S_{j}, \cdots, S_{n}\right\}$, where $S \subseteq \mathcal{S}$, the set of technological uncertain events. At aggregate level there are $\tilde{\mathcal{S}}=\{1, \cdots, \tilde{\mathcal{S}}\}$ states of nature. We count in total $(S+1)$ states of nature.

The economic agents are the $j \in\{1, \cdots, n\}$ producers and $i \in\{1, \cdots, m\}$ consumers which are characterized by sets of assumptions $F$ and $C$ bellow. There are $k \in\{1, \cdots, l\}$ physical commodities and $j \in\{1, \cdots, n\}$ financial assets, referred to as stocks. Physical goods are traded on each of the $(S+1)$ spot markets. Firms issue stocks which are traded at $s=0$, yielding a payoff in the next period at uncertain state $s \in\{1, \cdots, S\}$. The quantity vector of stocks issued by firm $j$ is denoted $Z_{j} \in \mathbb{R}_{-}$. Other assets such as bonds or options can be introduced without any further difficulties. There are total $l(S+1)$ goods. The consumption bundle of agent $i$ is denoted $x_{i}=\left(x_{i}(0), x_{i}(s), \cdots, x_{i}(S)\right) \in \mathbb{R}_{++}^{l(s+1)}$, with $x_{i}(s)=\left(x_{i}^{1}(s), \cdots, x_{i}^{l}(s)\right) \in \mathbb{R}_{++}^{l}$, and $\sum_{i=1}^{m} x_{i}=x$. The consumption space for each $i$ is $X_{i}=\mathbb{R}_{++}^{l(s+1)}$, the strictly positive orthant. The associated price system is a collection of vectors represented by $p=(p(0), p(s), \cdots, p(S)) \in \mathbb{R}_{++}^{l(S+1)}$, with $p(s)=\left(p^{1}(s), \cdots, p^{l}(s)\right) \in \mathbb{R}_{++}^{l}$. There are $n$ financial assets traded in period 0 . Denote the quantity vector of stocks purchased by consumer $i$, $z_{i}=\left(z_{i}(1), \cdots, z_{i}(j), \cdots, z_{i}(n)\right) \in \mathbb{R}_{+}^{n}$, and denote $\sum_{i=1}^{m} z_{i}=z$, with associated spot price system $q=(q(1), \cdots, q(j), \cdots, q(n)) \in \mathbb{R}_{++}^{n}$. We assume $l(S+1)$ complete commodity markets and model producers' sequential optimization behavior in an incomplete financial markets environment. Incomplete markets is shown to be a consequence of the technological uncertainty hypothesis. Denote producer $j$ s long run net activity vector

$$
y_{j}=\left(y_{j}^{m}(s) \times y_{j}^{n}(s), \cdots, y_{j}^{m}(s) \times y_{j}^{n}(s)\right) \in \mathbb{R}^{l s}
$$

where $y_{j}^{m}(s) \in \mathbb{R}_{-}^{m}$ represents the long run input vector and $y_{j}^{n}(s) \in \mathbb{R}_{+}^{n}$ the associated feasible output vector. A state $s$ net activity of the firm $j$ is denoted

$$
y_{j}(s)=\left(y_{j}^{1}(s), \cdots, y_{j}^{l}(s)\right) \in \mathbb{R}^{l}
$$

where by convention an element $y_{j}^{k}<0$ denotes a factor of production and $y_{j}^{k} \geq 0$ a good produced. Let $\sum_{j=1}^{n} y_{j}=y$ denote the long run net activity vectors.

Sequential behavior of the producers: Consider the sequential structure of the optimization problem of the firm. Firms build up long run production capacity in the first period, for that, they issue stocks. The value of total stocks issued in period one, denoted $q z_{j}=m_{j}$, where $m_{j} \in \mathbb{R}$ is a real number, bounds the quantity of goods a producer $j$ can buy in state $s \in S$ at input prices $p_{I N P}(s)$ in period two. Once money is received through financial 
markets, firms install production capacity, and production activities take place subject to constraint long run production sets in the second period. Uncertainty in production is introduced by a random variable $s \in S_{j}$ for every $j$.

Assumption (T): For every production set $Y_{j}(s), s \in S_{j} \geq 2$.

Assumption (P): Firms maximize long run profits.

Assumptions (F): (i) For each $j,\left.Y_{j}\right|_{z} \subset \mathbb{R}^{l s}$ is closed, convex, and $\left(\omega+\left.\sum_{j=1}^{n} Y_{j}\right|_{z}\right) \cap \mathbb{R}_{+}^{I S}$ compact $\forall \omega_{i} \in \mathbb{R}_{++}^{I S} .\left.\left.\quad 0 \in Y_{j}\right|_{z} \Leftarrow Y_{j}\right|_{z} \supset \mathbb{R}_{-}^{I S}$. $\left.Y_{j}\right|_{z} \cap \mathbb{R}_{+}^{I S}=\{0\}$. (ii) For each $j$, denote $\left.\partial Y_{j}\right|_{z} \subset \mathbb{R}^{n S}$ a $C^{\infty}$ manifold for transformation maps (1) $\phi_{j}: \mathbb{R}_{-}^{m} \times \mathbb{R}_{-}^{n} \rightarrow \mathbb{R}_{+}^{l}$ non-linear for all $s \in S^{1}$.

We replace the non-linearity assumption (1) in F with $F(2)$. Imposing linearity on the technology of the firm enables the modelling of firms which exhibit constant return to scales.

Assumption $\mathrm{F}(2): \quad \phi_{j}: \mathbb{R}_{-}^{m} \times \mathbb{R}_{-}^{n} \rightarrow \mathbb{R}_{+}^{l}$ for all $s \in S$ piecewise linear $\forall j$.

Many economic applications deal with linear activity models. We therefore, consider the case of linear technologies. In order to apply previous existence proof to these models, we need to regularize the convex, piece-wise linear production manifolds $\left.Y_{j}\right|_{\bar{z}}=\mathbb{R}^{n S}$ by convolution and show that these convolutes, denoted $\Phi_{j}$, are compact and smooth manifolds approximating the piecewise linear production manifolds. For that, we define the state dependent convolute for firm $j$

$$
\left(\lambda_{\sigma} * \phi_{j}(y)\right)_{j}(s)=\left\{\begin{array}{ll}
\int_{\mathbb{R}_{-}^{m}}\left(\lambda_{\sigma}(\zeta) \phi_{j}(y-\zeta) \mathrm{d} \zeta\right)_{j}(s) & \text { for } y \in U_{\sigma} \\
0 & \text { otherwise }
\end{array} \forall s, j\right.
$$

where $y \in U_{\sigma}$, and $U_{\sigma}=\{y \in U: B(y, \sigma) \subset U\}$. Continuity of $\phi_{j}(s)$ implies the existence of a distance $\sigma=\inf _{t}\left(\sigma_{t}\right)$, where $0<\sigma<1$. Associate with measure $\sigma \in[0,1]$ the manifolds $\lambda_{\sigma}$ defined by

$$
\lambda_{\sigma}(y)(s)=\frac{1}{\sigma} \lambda\left(\frac{y}{\sigma}\right)(s), \forall s
$$

Proposition 1. Each regularized manifold $\left.\partial \tilde{Y}_{j}\right|_{z}$ defined by the convolute $\Phi_{j}(s), \forall s$, is $C^{\infty}$ and compact.

Proof. For each $j$, denote the state dependent convolute

$$
\Phi(s)_{j}=(\lambda * \phi(y))_{j}(s)=\int_{\mathbb{R}_{-}^{m}}\left(\phi(y-\zeta)_{j} \lambda_{\sigma}(\zeta) \mathrm{d} \zeta\right)_{j}(s)
$$

Can restrict domain of integration to Int $\operatorname{supp}(\lambda)$. See (Dieudonnè [8]). Let $\lim _{p \rightarrow 0} y^{p}=-\infty$, and let $\lim _{p \rightarrow \infty} y^{p}=0$. Denote $A=(\{-\infty\}, 0)^{m} \subseteq \mathbb{R}_{-}^{m}$. For any $z \in \mathbb{R}_{+}^{n},\left.\exists y\right|_{z} \in A$. Denote the compact subset associated with any $z,\left.A\right|_{z}$. $\left.A\right|_{z} \subseteq A$. Then the image of the continuous map $\Phi:\left.\left.A\right|_{z} \rightarrow \partial \tilde{Y}\right|_{z}$ is compact by surjectivity of $\Phi$.

Proposition 2. For any $j$ and $C^{\infty}$ kernel $\lambda, \lambda *$ is bounded and converges to identity $\phi$, it satisfies $\left|\left(\lambda_{\sigma} * \phi\right)_{j}(s)-\phi(s)\right|_{j} \leq \varepsilon(s)_{j} \quad \forall s$.

${ }^{1}$ Here, $C^{\infty}$ implies differentiability at any order required. The order depends on all transversality arguments employed. $\mathrm{m}$ denotes the inputs and $\mathrm{n}$ the output elements of the production set, and $\mathrm{l}=$ $\mathrm{m}+\mathrm{n}$. 
Proof. Define for every $s \in S \operatorname{diam}(\lambda)$ with $\operatorname{supp}(\lambda)$ contained in the unit ball $\mathbb{R}_{-}^{m}$. Let $\varepsilon(s)=y(\phi \text {, } \operatorname{diam}(\lambda))_{j}(s)$. Now, for any $C^{\infty}$ kernel $\lambda$ can define $\phi$ in $\mathbb{R}^{l s}$ such that for all $s \in S$

$$
\left(\left(\lambda * \phi_{j}-\phi\right)(y)\right)_{j}(s)=\int_{\mathbb{R}^{m}}\left[\left(\phi(y-\zeta)_{j}-\phi(y)\right) \lambda(\zeta)^{\frac{1}{2}} \mathrm{~d} \zeta\right]_{j}(s),
$$

by Cauchy inequality and Fubini's theorem, and since mass of $\lambda$ is equal to one, and $\zeta$ ranges over its support, we obtain

$$
\left(\int_{\mathbb{R}_{-}^{m}}\left|\left(\lambda * \phi_{j}-\phi\right)(y)\right|^{2} \mathrm{~d} y\right)_{j}(s) \leq \sup _{\| \zeta \mid \leq \sigma}\left(\int_{\mathbb{R}_{-}^{m}}\left|\left(\phi(y-\zeta)_{j}-\phi(y)\right)\right|^{2} \mathrm{~d} y\right)_{j}(s)
$$

Thus it follows that

$$
\left(\int_{\mathbb{R}_{-}^{m}}\left|\left(\lambda * \phi_{j}-\phi\right)(y)\right| \mathrm{d} y\right)_{j}(s) \leq \sup _{\|\zeta\| \leq \sigma}\left(\left.\int_{\mathbb{R}_{-}^{m}}\left(\phi(y-\zeta)_{j}-\phi(y)\right)\right|^{2} \mathrm{~d} y\right)_{j}^{1 / 2}(s)
$$

denoted $y(\phi, \operatorname{diam}(\lambda))_{j}(s)$. It converges to zero when $\operatorname{diam}(\lambda)$ converges to zero. It is bounded above since

$$
y\left(\phi, \operatorname{diam}(\lambda)_{j}\right)(s) \leq c\left(\sum_{k=1}^{m}\left|D^{k} \phi(y)\right|_{j}^{2}(s)\right)^{\frac{1}{2}}
$$

where $c=k_{1} \sigma . k_{1}$ is a constant of differentiation, and $\sigma$ a distance.

\section{Existence of Equilibria}

In this section we show existence of equilibria. The strategy of the proof is to show that a pseudo equilibrium exists and that every pseudo equilibrium is also a financial markets equilibrium with production. It is known that pseudo equilibria exists for exchange economies. See Duffie, Shafer, Geanokopolos, Hirsh, Husseini, and others [9]-[14]. Genakopolos et al. [4] showed that pseuedo equilibria exist for an economy with production for the case of exogenous financial markets. At variance with their model, where the firm's problem is to solve a Nash equilibrium, we show that a pseudo equilibrium for a more general price and technology dependent asset structure, permitting the modeling of production and its finance, exists Stiefenhofer [7].

We prove the follwing result.

Theorem 1 (Existence Equilibria). Let the assumptions $T, P, F, F(2)$, and $C$ hold. Then for any $\left.\partial \tilde{Y}_{j}\right|_{\bar{z}}$, there exists a pseudo $F E$ with production $(\bar{x}, \bar{y}),(\bar{P}, \bar{L}) \in \mathbb{R}_{++}^{l(S+1) m} \times \mathbb{R}_{+}^{l(S+1) n} \times \mathfrak{S}^{\prime} \times G^{n}\left(\mathbb{R}^{S}\right)$ for generic endowments. Moreover, by the relational propositions a $F E$ with production $(\bar{x}, \bar{y}, \bar{z}),(\bar{p}, \bar{q}) \in \mathbb{R}_{++}^{l(S+1) m} \times \mathbb{R}_{+}^{l(S+1) n} \times \mathbb{R}^{n m} \times \mathfrak{S} \times \mathbb{R}_{++}^{n}$ exists for generic endowments.

Proof. Production takes place in the second period, once capacity is installed and state $s \in S$ occurred. At $t=0$, firms choose $z_{j}$ at price $q$ such that long run profits are maximized in every state $s \in S$ subject to long run technological feasibility $\phi_{j}$ and capacity constraints $m_{j}$. Denote the long run production set $\left.Y_{j}\right|_{z}$. This set is not independent of the firm's technology nor on 
its financial activities, denoted $Z$. More formally, the firm's sequential optimization problem is

$$
(\bar{z}, \bar{y})_{j} \arg \max \left\{\bar{p}(s) \square y_{j}(s): \mid \begin{array}{l}
\left.y_{j} \in Y_{j}\right|_{z} \\
\bar{q} z_{j}=\left[p(s) \cdot y_{j}(s)\right]_{I N P} \forall s \in S
\end{array}\right\} .
$$

Denote a long run equilibrium output vector associated with the production set boundary $\bar{y}_{j} \in \partial Y_{j}$,eff $\left.\right|_{2}$. Each firm $j$ is characterized by set of assumptions $F$ (Debreu [1]). We modify Debreu's assumptions on production sets in order to allow the modeling of endogenous production capacity via financial assets. The $t=1$ maps implied by Equation (1), $\pi_{j}: \mathbb{R}_{++}^{l} \times \mathbb{R}^{l} \times \mathbb{R}_{+} \rightarrow \mathbb{R}_{+}$, for each state $s \in S$ and all producers $j$ define the $(S \times n)$ total long run payoff matrix, a collection of $n$ vectors denoted

$$
\Pi\left(p_{1},\left.\phi\right|_{Z}\right)=\left[\begin{array}{ccc}
p(s) \cdot y_{1}(s) & \cdots & p(s) \cdot y_{n}(s) \\
\vdots & & \vdots \\
p(s) \cdot y_{n}(s) & \cdots & p(s) \cdot y_{n}(s)
\end{array}\right],
$$

where $\left.\phi\right|_{Z}$ denotes the technology and capacity dependency of the payoff structure. We next introduce the consumer side of the economy.

The consumer: Each consumer $i \in\{1, \cdots, m\}$ is characterized by set of assumptions $\mathrm{C}$ of smooth economies.

Assumptions (C): (i) $u_{i}: \mathbb{R}_{+}^{l(S+1)} \rightarrow \mathbb{R}$ is continuous on $\mathbb{R}_{+}^{l(S+1)}$ and $C^{\infty}$ on $\mathbb{R}_{++}^{l(S+1)}$.

$$
u_{i}\left(x_{i}\right)=\left\{x_{i}^{\prime} \in \mathbb{R}_{+}^{l(s+1)}: u_{i}\left(x_{i}^{\prime}\right) \geq u_{i}\left(x_{i}\right)\right\} \subset \mathbb{R}_{++}^{l(s+1)}, \forall x_{i} \in \mathbb{R}_{++}^{l(s+1)} .
$$

For each $x_{i} \in \mathbb{R}_{++}^{l(S+1)}, D u_{i}\left(x_{i}\right) \in \mathbb{R}_{++}^{l(S+1)}, \forall s$. For each $x_{i} \in \mathbb{R}_{++}^{l(S+1)}$, $h^{\mathrm{T}} D^{2} u_{i}\left(x_{i}\right) h<0$, for all nonzero hyperplane $h$ such that $\left(D u_{i}\left(x_{i}\right)\right)^{\mathrm{T}} h=0$. (ii) Each $i$ is endowed with $\omega_{i} \in \mathbb{R}_{++}^{l(S+1)}$.

Consumers want to transfer wealth between future spot markets. For that, they invest in firms in period $t=0$, receiving a share of total dividend payoffs which are determined in the next period in return. Denote the sequence of $(S+1)$ budget constraints

$$
B_{z_{i}}=\left\{x_{i} \in \mathbb{R}_{++}^{l(s+1)}, z_{i} \in \mathbb{R}_{+}^{n}: \begin{array}{l}
p(0) \cdot\left(x_{i}(0)-\omega_{i}(0)\right)=-q z_{i} \\
p(s) \square\left(x_{i}(s)-\omega_{i}(s)\right)=\Pi\left(p_{1}, \phi\right) \theta\left(z_{i}\right)
\end{array}\right\},
$$

where ${ }^{2}$ ownership structure is a $(n \times 1)$ vector defined by the mappings

$$
\theta_{i j}: \mathbb{R}_{+} \rightarrow \mathbb{R}_{+}, \forall j,
$$

where $Z_{i}(j) \in \mathbb{R}_{+}$is a positive real number for every $j=1, \cdots, n$.

$\theta_{i j}=z_{i}(j)\left[\sum_{i} z_{i}(j)\right]^{-1}$ is the proportion of total payoff of financial asset $j$ hold by consumer $i \in I$. In compressed notation, we write

$$
B_{z_{i}}=\left\{x_{i} \in \mathbb{R}_{++}^{l(s+1)}, z_{i} \in \mathbb{R}_{+}^{n}: p(s) \square\left(x_{i}(s)-\omega_{i}(s)\right) \in \hat{\Pi}\left[z_{i} \mid \theta\left(z_{i}\right)\right]\right\}
$$

\footnotetext{
${ }^{2} \square$ denotes the box product. A "s by s" context dependent mathematical operation. For example the $s$ by $s$ inner product.
} 
where $\hat{\Pi}\left(p_{1}, q, y\right)=\left[\begin{array}{ccc}-q_{1} & \cdots & -q_{n} \\ p(1) \cdot y_{1}(1) & \cdots & p(1) \cdot y_{n}(1) \\ \vdots & & \vdots \\ p(S) \cdot y_{1}(S) & \cdots & p(S) \cdot y_{n}(S)\end{array}\right]$ represents the full pay-

off matrix of order $((S+1) \times n)$.

We introduce following prize normalization $\mathfrak{S}=\left\{p \in \mathbb{R}_{++}^{l(S+1)}:\|p\|=\Delta\right\}$ such that the Euclidean norm vector of the spot price system $\|p\|$ is a strictly positive real number $\Delta \in \mathbb{R}_{++}$.

Definition 1. A financial markets equilibrium with production $(\bar{x}, \bar{y}, \bar{z}),(\bar{p}, \bar{q}) \in \mathbb{R}_{++}^{l(S+1) m} \times \mathbb{R}_{+}^{l(S+1) n} \times \mathbb{R}^{n m} \times \mathfrak{S} \times \mathbb{R}_{++}^{n}$ satisfies.

(i) $\left(\bar{x}_{i} ; \bar{z}_{i}\right) \arg \max \left\{u_{i}\left(x_{i} ; z_{i}\right): \bar{x}_{i} \in B_{\bar{z}_{i}}\left(\bar{p}, \bar{q}, \bar{y} ; \omega_{i}\right)\right\} \quad \forall i$

(ii) $(\bar{z}, \bar{y})_{j} \arg \max \left\{\bar{p}(s) \square y_{j}(s): \mid \begin{array}{l}\left.y_{j} \in Y_{j}\right|_{z} \\ \bar{q} z_{j}=\left[\bar{p}(s) \cdot y_{j}(s)\right]_{I N P} \quad \forall s \in S\end{array}\right\} \forall j$

(iii) $\sum_{i}^{m}\left(\bar{x}_{i}-\omega_{i}\right)=\sum_{j}^{n} \bar{y}$

(iv) $\sum_{i=1}^{m} \theta\left(\bar{z}_{i}\right)=1 \forall j$, and $\sum_{j=1}^{n} \sum_{i=1}^{m}\left(\bar{z}_{i}\right)_{j}=1=0$.

(i) and (ii) are the optimization problems of the consumers and producers. (iii) and (iv) represent physical goods and financial markets clearance conditions. $\sum_{i=1}^{m} \theta\left(\bar{z}_{i}\right)_{j}=1 \forall j$ states that each firm $j$ is owned by the consumers.

Definition 2. if $\nexists z \in \mathbb{R}_{++}^{n}$ s.t.

$\hat{\Pi}\left(p_{1}, q, \phi\right)\left[z \mid \sum_{i=1}^{m} \theta\left(z_{i}\right)_{s=1}^{S}\right] \geq 0$, then $q \in \mathbb{R}_{++}^{n}$ is a no-arbitrage asset price relative to $p_{1}$.

Lemma 1. $\exists \beta \in \mathbb{R}_{++}^{S}$ s.t. $q=\sum_{s=1}^{S} \beta \square \Pi\left(p_{1}, \phi\right)$.

Proof. Immediate consequence of the separation theorem for $((S+1) \times n)$ matrices in Gale (1960). It asserts that either $\exists z \in \mathbb{R}_{++}^{n}$ such that $\hat{\Pi} z \geq 0$, or $\exists \beta \in \mathbb{R}_{++}^{S+1}$ such that $\beta \hat{\Pi}=0$.

We can now rescale equilibrium prices without affecting equilibrium allocations, let $P_{1}=\beta \square \bar{p}_{1}$. The next step is to derive a normalized no arbitrage equilibrium definition [15]. Let $\beta \in \mathbb{R}_{++}^{s}$ be $\left(\frac{\lambda(s)}{\lambda}\right)_{i=1}$ the gradient vector from the optimization problem of agent 1 , called the Arrow-Debreu agent. The Walrasian budget set for the Arrow-Debreu agent is a sequence of constraints denoted

$$
B_{1}=\left\{\begin{array}{c}
P \cdot\left(x_{i}-\tilde{\omega}_{i}\right)=0 \\
x_{1} \in \mathbb{R}_{++}^{l(s+1)}: P(s) \square\left(x_{i}(s)-\omega_{i}(s)\right)=\sum_{j} \theta_{i j} P(s) \cdot y_{j}(s)
\end{array}\right\} .
$$

For all consumers $i \geq 2$, the no arbitrage budget set consisting of a sequence of $(S+1)$ constraints is denoted

$$
B_{i \geq 2}=\left\{x_{i} \in \mathbb{R}_{++}^{l(s+1)}: \begin{array}{l}
P \cdot\left(x_{i}-\tilde{\omega}_{i}\right)=0 \\
P(s) \square\left(x_{i}(s)-\omega_{i}(s)\right) \in\left\langle\Pi\left(P_{1}, \phi\right)\right\rangle
\end{array}\right\},
$$

where $\left\langle\Pi\left(P_{1}, \phi\right)\right\rangle$ is the span of the income transfer space of period one. Re- 
place $\left\langle\Pi\left(P_{1}, \phi\right)\right\rangle$ with $L$ in $G^{n}\left(\mathbb{R}^{S}\right)$, where $G^{n}\left(\mathbb{R}^{S}\right)$ is the Grassmann manifold ${ }^{3}$ with its known smooth $(S-n) n$ dimensional structure, and $L$ an $n$ dimensional affine subspace of $G^{n}\left(\mathbb{R}^{S}\right)$.

Denote the pseudo opportunity set $B_{i}\left(P, L ; \omega_{i}\right)$, for each $i$,

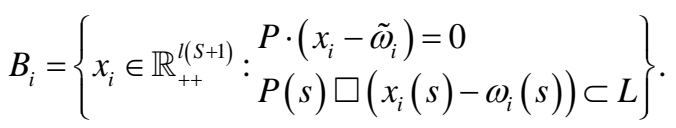

Let $\mathfrak{S}^{\prime}=\left\{p \in \mathbb{R}_{++}^{l(S+1)}: p^{0,1}=\Delta\right\}$ be the set of normalized prices, and let $\Delta \in \mathbb{R}_{++}$be a fixed strictly positive real number. This convenient normalization singles out the first good at the spot $s=0$ as the numeraire. We introduce following definitions for the long run payoff maps associated with sets $\mathfrak{S}$ and $\mathfrak{S}^{\prime}$ :

Definition 3. (i) For any $p_{1} \in \mathfrak{S}$, such that $\pi: \mathfrak{S} \times \mathbb{R}^{l} \times \mathbb{R}_{+} \rightarrow \mathcal{A}$, let

$$
\Gamma\left(P_{1}, \phi\right)=\beta \square\left[\left(\operatorname{proj}_{\Delta}\left(\frac{1}{\beta}\right)^{\mathrm{T}} \square P_{1}\right) \square y\right]
$$

where $\mathrm{T}$ denotes the transpose,

$$
\operatorname{proj}_{\Delta}(z)=\Delta\left(\frac{z}{\|z\|}\right), \frac{1}{\beta}=\left(\frac{1}{\beta(1)}, \cdots, \frac{1}{\beta(S)}\right) \in R_{++}^{S}
$$

and

$$
\beta=(\beta(1), \cdots, \beta(S)) \in R_{++}^{S}
$$

(ii) For any $p_{1} \in \mathfrak{S}^{\prime}$, such that $\pi: \mathfrak{S}^{\prime} \times \mathbb{R}^{l} \times \mathbb{R}_{+} \rightarrow \mathcal{A}$, let

$$
\Gamma\left(P_{1}, \phi\right)=\beta \square\left[\left(\left(\frac{1}{\beta}\right)^{\mathrm{T}} \square P_{1}\right) \square y\right]
$$

where $\mathcal{A}$ is a set of $(S \times n)$ matrices $A$ of order $(S \times n)$.

We can now define the pseudo financial markets equilibrium with production. We then state the relational propositions between a full rank FE with production and a pseudo $\mathrm{FE}$ with production.

Definition 4. A pseudo financial markets equilibrium with production $(\bar{x}, \bar{y}),(\bar{P}, \bar{L}) \in \mathbb{R}_{++}^{l(S+1) m} \times \mathbb{R}_{+}^{l(S+1) n} \times \mathfrak{S}^{\prime} \times G^{n}\left(\mathbb{R}^{S}\right)$ satisfies:

(i) $\left(\bar{x}_{1}\right) \arg \max \left\{u_{1}\left(x_{1}\right)\right.$ s.t. $\left.x_{1} \in B_{1}\left(\bar{P}, \omega_{1}\right)\right\} \quad i=1$

(ii) $\left(\bar{x}_{i}\right) \arg \max \left\{u_{i}\left(x_{i}\right)\right.$ s.t. $\left.x_{i} \in B_{i}\left(\bar{P}, \bar{L}, \omega_{i}\right)\right\} \forall i \geq 2$

(iii) $\left\langle\Gamma\left(\bar{P}_{1}, \bar{\phi}\right)\right\rangle \subset \bar{L}$, proper if $\left\langle\Gamma\left(\bar{P}_{1}, \bar{\phi}\right)\right\rangle=\bar{L}$

(iv) $(\bar{y})_{j} \arg \max \left\{\bar{p}(s) \square y_{j}(s):\left\{\begin{array}{l}\left.y_{j} \in Y_{j}\right|_{z} \\ \bar{m}_{j}=\left[\bar{p}(s) \cdot y_{j}(s)\right]_{I N P} \quad \forall s \in S\end{array}\right\} \forall j\right.$

(v) $\bar{x}_{1}+\sum_{i=2}^{m} \bar{x}_{i}=\sum_{i=1}^{m} \omega_{i}+\sum_{j=1}^{n} \bar{y}_{j}$

Stiefenhofer [7] shows that for every full rank FE with production $(\bar{x}, \bar{y}, \bar{z}),(\bar{p}, \bar{q})$, there exists $\beta \in \mathbb{R}_{++}^{S}$ and a $n$-dimensional subspace $L \in G^{n}\left(\mathbb{R}^{S}\right)$ ${ }^{3}$ See i.e. Dieudonnè [8] for properties of the Grassmann manifold. See Duffie and Shafer for an exposition of the Grassmann manifold in economics [9]. 
such that $(\bar{x}, \bar{y}),(\bar{P}, \bar{L})$ is a pseudo $\mathrm{FE}$ with production. Moreover, if $(\bar{x}, \bar{y}),(\bar{P}, \bar{L})$ is a pseudo $\mathrm{FE}$ with production then for every $\beta \in \mathbb{R}_{++}^{S}$, there exist financial asset prices $\bar{q} \in \mathbb{R}_{++}^{n}$ and investment portfolios

$\bar{z}=(z(1), \cdots, z(n)) \in \mathbb{R}_{++}^{n}$ such that $(\bar{x}, \bar{y}, \bar{z}),(\bar{p}, \bar{q})$ is a $(\bar{x}, \bar{y})$ allocational equivalent $\mathrm{FE}$ with production.

Long run financial payoffs depend on the technology of the firm, its production capacity installed via financial markets, and on a set of regular prices. Equilibrium does not exist for critical prices. The next step is therefore to introduce rank dependant payoff maps, and to exhibit a class of transverse price, technology, and capacity dependent maps. We will show that equilibria exists for this smooth rank dependent real asset structure, denoted $\pi^{\rho}$

Definition 5. Define the rank dependent long run payoff maps $\pi^{\rho}: \mathbb{R}_{++}^{l} \times \mathbb{R}^{l} \times \mathbb{R}_{+} \rightarrow \mathcal{A}^{\rho}$ for $0 \leq \rho \leq n$. The set of reduced rank matrices $A^{\rho}$ of order $(S \times n)$ with $\operatorname{rank}\left(A^{\rho}\right)=(n-\rho)$ is denoted $\mathcal{A}^{\rho}$ and is of order $(S \times n)$.

The following properties are well known. (i) For $1 \leq \rho<n, A^{\rho}$ is a submanifold of $A$ of codimension $(S-n+\rho) \rho$. (ii) for $\rho=n$ the set $\mathcal{A}^{\rho}=\{\varnothing\}$ is empty, and (iii) for $\rho=0, \mathcal{A}^{\rho}=\mathcal{A}$ the set of reduced rank matrices is equivalent to the set of full rank matrices. These properties states that, for $1 \leq \rho<n$, the incomplete income transfer space is rank reduced. The rank dependent endogenized long run asset structure has following properties.

Lemma 2. (i) $\pi^{\rho} \pitchfork \mathcal{A}^{\rho}$ for integers $1 \leq \rho \leq n$. (ii) $\Gamma^{\rho} \pitchfork \mathcal{A}^{\rho}$ for any $\beta \in \mathbb{R}_{++}^{S}$ and integers $1 \leq \rho \leq n$. (iii) $\Gamma^{\rho} \cap \mathcal{A}$ is generic, since it is dense and open.

Proof. (i) The linear map $D_{y} \pi^{\rho}$ is surjective everywhere in $Y$. (ii) This property does not change for any $\beta \in \mathbb{R}_{++}^{S}$. (iii) Immediate consequence of the transversality theorem for maps. Since each set $\pitchfork\left(\Gamma^{\rho}, \mathcal{A} ; \mathcal{A}^{\rho}\right)$ is residual, their intersection is residual.

Definition 6. Denote $\Psi^{\rho}$ the vector bundle defined by (i) a basis

$$
P^{\rho}=\left\{P \in \mathbb{R}_{++}^{l(S+1)}: \operatorname{rank}\left(\Gamma^{\rho}\left(P_{1}, \phi\right)\right)=(n-\rho)\right\},
$$

and (ii) orthogonal income transfer space $L^{\perp} \subset\left\langle\Gamma^{\rho}\left(P_{1}, \phi\right)\right\rangle^{\perp}$,

$$
\Psi^{\rho}=\left\{\left(P,\left\langle\Gamma^{\rho}\left(P_{1}, \phi\right)\right\rangle^{\perp}, L^{\perp}\right) \in P^{\rho} \times G^{S-n+\rho}\left(\mathbb{R}^{S}\right) \times G^{S-n}\left(\mathbb{R}^{S}\right): L^{\perp} \subset\left\langle\Gamma^{\rho}\left(P_{1}, \phi\right)\right\rangle^{\perp}\right\} .
$$

We thus have defined a fiber bundle $\Psi^{\rho}$ of codimension $l(S+1)-1-\rho^{2}$ containing the spot price system and income transfer space consisting of a base vector $P^{\rho}$ and fiber $G^{S-n}\left(\mathbb{R}^{S-n+\rho}\right)$.

By propositions (1) and (2) the piecewise linear production manifolds are regularized by convolution and are approximated smooth manifolds. Then, it follows that by lemma (2) and using definitionn (6) we can define an evaluation map $Z^{\rho}$ on $\Psi^{\rho} \times \mathbb{R}_{++}^{l(S+1) m}$, where we denote by $\Omega=\mathbb{R}_{++}^{l(S+1) m}$ the set of the economy's total initial endowments, such that the excess demand map $Z^{\rho}: \Psi^{\rho} \times \Omega \rightarrow N$.

For the Arrow-Debreu agent have 


$$
Z_{1}^{\rho}: \Psi^{\rho} \times \Omega \rightarrow N
$$

The evaluation map is a submersion, since $D_{\omega_{1}} Z_{1}^{\rho} \forall \omega_{1} \in \Omega$ is surjective everywhere. $\exists$ for each $\omega_{1} \in \Omega$

$$
Z_{1, \omega_{1} \in \Omega}^{\rho}: \Psi^{\rho} \rightarrow N \pitchfork_{\omega \in \Omega}\{0\},
$$

where $\{0\} \subset N$, and $\rho=0$. The dimension of the preimage $Z_{1, \omega_{1} \in \Omega}^{-1}(\{0\})$ is $l(S+1)-1$. By Thom's parametric transversality theorem ${ }^{4}$, it follows that the subset $\Omega_{\rho} \cap \Omega$ is generic since it is open and dense. Equilibria exist. By the equivalence propositions 2 and 3 know that full rank financial markets equilibria with production exist.

For all $1 \leq \rho \leq n$ the preimage of the rank reduced evaluation map has dimension $l(S+1)-1-\rho^{2}$. This implies that for generic endowments $\omega \in \bigcap_{\rho}\left(\Omega_{\rho}\right)$, for $\rho=1, \cdots, n$, there is no reduced rank equilibrium, since for $Z_{1}^{\rho}(., \omega)$ the set of $\{0\}=\varnothing$.

\section{Conclusion}

The paper discusses a model of production when production risks cannot be fully hedged. It formulates a general equilibrium model where production capacity is financed through financial markets and production costs are financed through the firm's revenue. The main novelty of the model is the way production is financed. At variance to the literature, the model considered in this paper introduces an objective function of the firm, which is independent of any utility of a shareholder. This property rehabilitates the decentralization property of the Arrow-Debreu model. The central object of study in this paper is the firm with its linear technology exhibiting constant returns to scale. Assuming linear technologies leads to the mathematical problem of proving existence of equilibria. It is shown that production sets, defined by convex cones, can be regularized by convolution leading to approximately smooth manifolds. This is a useful result, since techniques of differential topology can now be applied to a study of the economic properties of the model. In this paper, we show that equilibria exists. Future research should explore the set of solutions of this model in much greater depth. If it can be shown that the set of solutions forms a smooth manifold then many properties of the model can be extracted by applying techniques from differential topology. This is work in progress.

\section{References}

[1] Debreu, G. (1959) Theory of Value. Wiley, New York.

[2] Drèze, J.H. (1974) Investment under Private Ownership: Optimality, Equilibrium and Stability. In: Drèze, J.H., Ed., Allocation under Uncertainty: Equilibrium and Optimality, Wiley, New York, 129-165. https://doi.org/10.1007/978-1-349-01989-2_9

${ }^{4}$ See i.e. Hirsch for an exposition of Thom's parametric transversality theorem [16]. For more on transversality see R. Abraham and J. Robbin (1967), Transversal Mappings and Flows. (W.A. Benjamin). 
[3] Hart, O. (1975) On the Optimality of Equilibrium When the Market Structure Is Incomplete. Journal of Economic Theory, 11, 418-443. https://doi.org/10.1016/0022-0531(75)90028-9

[4] Geanokopolos, J., Magill, M., Quinzii, M. and Drèze, J. (1990) Generic Inefficiency of Stock Market Equilibrium When Markets Are Incomplete. Journal of Mathematical Economics, 19, 113-151. https://doi.org/10.1016/0304-4068(90)90039-C

[5] Ekern, S. and Wilson, R. (1974) On the Theory of the Firm in an Economy with Incomplete Markets. Bell Journal of Economics and Management Science, 5, 171-180. https://doi.org/10.2307/3003097

[6] Grossman, S. and Hart, O. (1979) A Theory of Competitive Equilibrium in Stock Market Economies. Econometrica, 47, 293-330. https://doi.org/10.2307/1914186

[7] Stiefenhofer, P. (2016) Production in General Equilibrium with Incomplete Finanical Markets. Journal of Mathematical Finance, 6, 293-302. https://doi.org/10.4236/jmf.2016.62025

[8] Dieudonnè, J. (1972) Elèments d'analyse. Gauthier-Villars, Paris.

[9] Duffie, D. and Shafer, W. (1985) Equilibrium in Incomplete Markets I: A Basic Model of Generic Existence. Journal of Mathematical Economics, 14, 285-300. https://doi.org/10.1016/0304-4068(85)90004-7

[10] Bottazzi, J. (1995) Existence of Equilibria with Incomplete Markets: The Case of Smooth Returns. Journal of Mathematical Economics, 24, 59-72. https://doi.org/10.1016/0304-4068(94)00672-W

[11] Geanokopolos, J. and Shafer, W. (1990) Solving a System of Simultaneous Equations in Economics. Journal of Mathematical Economics, 19, 69-95.

[12] Hirsch, M., Magill, M. and Mas-Colell, A. (1990) A Geometric Approach to a Class of Equilibrium Existence Theorems. Journal of Mathematical Economics, 19, 95106.

[13] Husseini, S.Y., Lasry, J.M. and Magill, M. (1990) Existence of Equilibrium with Incomplete Markets. Journal of Mathematical Economics, 19, 39-67.

[14] Magill, M. and Shafer, W. (1990) Incomplete Markets. In: Hildebrand, W. and Sonnenschein, H., Eds., Handbook of Mathematical Economics, Vol. 4, Chapter 30, 1523-1614.

[15] Cass, D. (1984) Competitive Equilibria with Incomplete Financial Markets. Journal of Mathematical Economics, 42, 384-405.

[16] Hirsch, M. (1972) Differential Topology. Graduate Texts in Mathematics. Springer Verlag, New York. 
Submit or recommend next manuscript to SCIRP and we will provide best service for you:

Accepting pre-submission inquiries through Email, Facebook, LinkedIn, Twitter, etc. A wide selection of journals (inclusive of 9 subjects, more than 200 journals)

Providing 24-hour high-quality service

User-friendly online submission system

Fair and swift peer-review system

Efficient typesetting and proofreading procedure

Display of the result of downloads and visits, as well as the number of cited articles Maximum dissemination of your research work

Submit your manuscript at: http://papersubmission.scirp.org/

Or contact jmf@scirp.org 